\title{
List circular backbone colouring
}

\author{
Frédéric Havet $\|$ and Andrew D. King $\|^{2}$ \\ ${ }^{1}$ Projet COATI, I3S (CNRS, UNSA) \& INRIA Sophia Antipolis, and Simon Fraser University, PIMS, UMI 3069, CNRS. \\ ${ }^{2}$ Departments of Mathematics and Computing Science, Simon Fraser University, Burnaby, BC, Canada.
}

received $17^{\text {th }}$ Jan. 2013, revised $20^{\text {th }} \mathrm{Feb} .2014$, accepted $26^{\text {th }} \mathrm{Feb} .2014$.

\begin{abstract}
A natural generalization of graph colouring involves taking colours from a metric space and insisting that the endpoints of an edge receive colours separated by a minimum distance dictated by properties of the edge. In the $q$ backbone colouring problem, these minimum distances are either $q$ or 1 , depending on whether or not the edge is in the backbone. In this paper we consider the list version of this problem, with particular focus on colours in $\mathbb{Z}_{p}-$ this problem is closely related to the problem of circular choosability.

We first prove that the list circular q-backbone chromatic number of a graph is bounded by a function of the list chromatic number. We then consider the more general problem in which each edge is assigned an individual distance between its endpoints, and provide bounds using the Combinatorial Nullstellensatz. Through this result and through structural approaches, we achieve good bounds when both the graph and the backbone belong to restricted families of graphs.
\end{abstract}

Keywords: backbone colouring, list colouring, Combinatorial Nullstellensatz, planar graph

\section{Introduction}

All graphs considered in this paper are simple. Let $G=(V, E)$ be a graph, and let $H=(V, E(H))$ be a subgraph of $G$, called the backbone. A $k$-colouring of $G$ is a mapping $f: V \rightarrow\{1,2, \ldots, k\}$. Let $f$ be a $k$-colouring of $G$. It is a proper colouring if $|f(u)-f(v)| \geq 1$. It is a $q$-backbone colouring for $(G, H)$ if $f$ is a proper colouring of $G$ and $|f(u)-f(v)| \geq q$ for all edges $u v \in E(H)$. The chromatic number $\chi(G)$ is the smallest integer $k$ for which there exists a proper $k$-colouring of $G$. The $q$-backbone chromatic number $\operatorname{BBC}_{q}(G, H)$ is the smallest integer $k$ for which there exists a $q$-backbone $k$-colouring of $(G, H)$.

If $f$ is a proper $k$-colouring of $G$, then $g$ defined by $g(v)=q \cdot f(v)-(q-1)$ is a $q$-backbone $(q \cdot k-q+1)$-colouring of $(G, H)$ for any spanning subgraph $H$ of $G$. Hence,

$$
\operatorname{BBC}_{q}(G, H) \leq q \cdot \chi(G)-q+1 .
$$

\footnotetext{
*Partly supported by ANR Blanc International GRATEL.

† Supported by a PIMS Postdoctoral Fellowship and the NSERC Discovery Grants of Pavol Hell and Bojan Mohar.
} 
In [4, 5], Broersma et al. showed that for any integer $k$ there is a graph $G$ with $\chi(G)=k$ and a spanning tree $T$ such that $\mathrm{BBC}_{2}(G, T)=2 k-1$.

One can generalize the notion of backbone colouring by allowing a more complicated structure of the colour space. A natural choice is to impose a circular metric on the colours. We can see $\mathbb{Z}_{k}$ as a cycle of length $k$ with vertex set $\{1, \ldots, k\}$ together with the graphical distance $|\cdot|_{k}$. Then $|a-b|_{k} \geq q$ if and only if $q \leq|a-b| \leq k-q$. A circular $q$-backbone $k$-colouring of $G$ or $q$-backbone $\mathbb{Z}_{k}$-colouring of $(G, H)$ is a mapping $f: V(G) \rightarrow \mathbb{Z}_{k}$ such that $c(v) \neq c(u)$ for each edge $u v \in E(G)$ and $|c(u)-c(v)|_{k} \geq q$ for each edge $u v \in E(H)$. The circular q-backbone chromatic number of a graph pair $(G, H)$, denoted $\operatorname{CBC}_{q}(G, H)$, is the minimum $k$ such that $(G, H)$ admits a circular $q$-backbone $k$-colouring.

A backbone $\mathbb{Z}_{k}$-colouring is trivially a backbone $k$-colouring. On the other hand, a backbone $k$ colouring yields a circular backbone $\mathbb{Z}_{k+q-1}$-colouring. Hence for every graph pair $(G, H)$ (where $H$ is a subgraph of $G$ ), we have

$$
\mathrm{BBC}_{q}(G, H) \leq \mathrm{CBC}_{q}(G, H) \leq \mathrm{BBC}_{q}(G, H)+q-1 .
$$

Inequalities 1 and 2 yield

$$
\mathrm{CBC}_{q}(G, H) \leq q \cdot \chi(G) \text { for every subgraph } H \text { of } G .
$$

This bound is tight if $H$ contains a complete graph of size $\chi(G)$. In particular, it is the case when $G$ is bipartite, and $H$ is non-empty. On the other hand, Broersma et al. [6] gave better upper bounds when the backbone is a matching. We explore similar restrictions shortly, but first we define a list colouring analogue. Such an analogue is motivated by the fact that backbone colouring models a special case of radio channel assignment: in such problem, because of technical reasons or dynamicity, the set of channels available (i.e. colours) very often varies from transmitter (i.e. vertex) to transmitter.

\subsection{List backbone colourings}

The notions of backbone and circular backbone colouring naturally generalize to list colouring. An $S$-list assignment of a graph $G$ is a mapping $L$ which assigns to each vertex $v \in V(G)$ a prescribed list of colours $L(v) \subseteq S$, where $S$ is a set of colours. For a list assignment $L$, an $L$-colouring is a colouring $c$ such that $c(v) \in L(v)$ for every vertex. The list chromatic number of a graph $G$, denoted $\chi^{\ell}(G)$, is the least integer $k$ such that for any list assignment $L$ such that $|L(v)| \geq k$, there is a proper $L$-colouring. The graph pair $(G, H)$ is said to be circularly $q$-backbone $k$-choosable if for any $\mathbb{Z}_{p}$-list assignment $L$ such that $|L(v)| \geq k$, there is an $L$-colouring that is a $q$-backbone $\mathbb{Z}_{p}$-colouring. The list circular $q$-backbone number of a pair $(G, H)$, denoted $\mathrm{CBC}_{q}^{\ell}(G, H)$ is the least integer $k$ such that $(G, H)$ is circularly $q$ backbone $k$-choosable.

The concept of circular choosability, introduced by Mohar [15] and Zhu [20], is closely related to list circular backbone colouring. Indeed, the circular list chromatic number or circular choice number of $G$ may be defined as

$$
\operatorname{cch}(G):=\inf \left\{\operatorname{CBC}_{q}^{\ell}(G, G) / q\right\} .
$$

Zhu [20] proved that $\operatorname{cch}(G) \geq \chi^{\ell}(G)-1$ for every graph $G$. This implies that $\operatorname{CBC}_{q}^{\ell}(G, G) \geq q \cdot \chi^{\ell}(G)-$ $q$ for every positive $q$. In view of this inequality and the fact that $\mathrm{CBC}_{q}(G, G)=q \cdot \chi(G)$, it is natural to ask whether $\operatorname{CBC}_{q}^{\ell}(G, G)$ can be bounded by a function of $\chi^{\ell}(G)$. We answer in the positive in Section 2 
However, the function we prove is exponential in $\chi^{\ell}(G)$ - we believe that it is far from optimal, and pose the following problem.

Problem 1 Let $q$ be a positive integer. What is the minimum function $m_{q}$ such that for every $k$-choosable graph $G, \mathrm{CBC}_{q}^{\ell}(G, G) \leq m_{q}(k)$ ? In other words, what is $m_{q}(k)=\max \left\{\mathrm{CBC}_{q}^{\ell}(G, G) \mid \chi^{\ell}(G)=k\right\}$ ?

This is closely related to a conjecture of Zhu [20].

Conjecture 2 (Zhu [20]) There is a constant $\alpha$ such that, for every graph $G, \operatorname{cch}(G) \leq \alpha \cdot \chi^{\ell}(G)$.

Note that if such an $\alpha$ exists then it is at least two, as for any positive integer $m, \chi^{\ell}\left(K_{k, m^{k}}\right) \leq k+1$ because $K_{k, m^{k}}$ is $k$-degenerate, and $\operatorname{cch}\left(K_{k, m^{k}}\right) \geq\left(2-\frac{2 k}{m}\right) k$ as shown by Zhu [20]. Conjecture 2 would be implied by the following.

Conjecture 3 Let $q$ be a positive integer. There is a constant $\alpha_{q}$ such that, for every graph $G, \mathrm{CBC}_{q}^{\ell}(G, G) \leq$ $\alpha_{q} \cdot q \cdot \chi^{\ell}(G)$

As an evidence in support of the latter conjecture, we prove (Theorem 17) that for a graph $G$ and a matching $M, \mathrm{CBC}_{2}^{\ell}(G, M) \leq 2 \cdot \chi^{\ell}(G)+1$ and $\mathrm{CBC}_{q}^{\ell}(G, M) \leq 2(1+o(1)) \chi^{\ell}(G)$, where $o(1)$ is a function tending to 0 when $\chi^{\ell}(G)$ tends to infinity. These bounds generalize to any backbone $H$ in an upper bound in terms of $\chi^{\ell}(G)$ and $\chi^{\prime}(H)$, the chromatic index of $H$ : $\operatorname{CBC}_{2}^{\ell}(G, H) \leq 2^{\chi^{\prime}(H)} \chi^{\ell}(G)+1$ and for any $q \geq 3, \mathrm{CBC}_{q}^{\ell}(G, H) \leq 2^{\chi^{\prime}(H)}(1+o(1)) \chi^{\ell}(G)$.

In Section 3] we consider list circular backbone colouring of 2-choosable graphs. In [7], Erdős, Rubin, and Taylor characterized such graphs as follows. The heart of a graph $G$ is the maximal subgraph in which there is no vertex of degree one. The graph consisting of two vertices connected with three internally vertex-disjoint paths of length $i, j$ and $k$ is $\theta_{i, j, k}$.

Theorem 4 (Erdös et al. [7]) A connected graph is 2-choosable if and only if its heart is either a single vertex, an even cycle or $\theta_{2 m, 2,2}$ for some integer $m \geq 1$.

Using this characterization, Havet et al. [10] proved that if $G$ is 2-choosable, then $\operatorname{CBC}_{q}^{\ell}(G, G) \leq$ $5 q / 2$. This upper bound was later improved by Norine, Wong, and Zhu [17] to $16 q / 7$. In other words, $m_{q}(2) \leq 16 q / 7$. They conjectured that $m_{q}(2)=2 q$.

Conjecture 5 (Norine, Wong, and Zhu [17]) If $G$ is 2 -choosable, then $\operatorname{CBC}_{q}^{\ell}(G, G)=2 q$.

This conjecture is trivial for trees and was proved by Norine [16] for even cycles (and so for all graphs whose heart is an even cycle).

Theorem 6 (Norine [16]) If $C$ is an even cycle, then $\mathrm{CBC}_{q}^{\ell}(C, C)=2 q$ for all $q$.

Norine, Wong, and Zhu [17] also verified Conjecture 5 for $K_{2,3}=\theta_{2,2,2}$.

Note that Conjecture 5 implies that if $G$ is 2-choosable, then $\mathrm{CBC}_{q}^{\ell}(G, H)=2 q$ for any non-empty subgraph $H$ of $G$. We prove that this is the case if $H$ is a matching (Theorem 28). We also show that if $H$ does not contain the heart of $G$, then $\operatorname{CBC}_{q}^{\ell}(G, H) \leq 2 q+1$. 


\subsection{Edge-weighted backbones}

In Section 4 we generalize the idea of a backbone colouring by giving every edge $e$ a positive integer weight $w(e)$ dictating the minimum distance between the colours of its endpoints. This corresponds to the general channel assignment problem (see e.g. [12, 13]), in which the minimum distance between the channels assigned to two transmitters depends on these transmitters and how they can interfere with each other. In this paper, we apply the technique developed in this section to prove a result on list circular backbone colouring. However, as the technique is quite general, we believe that it could be used on other channel assignment problems.

Formally, a colouring $c$ of $G$ of $G$ is $w$-coherent if $|c(u)-c(v)| \geq w(u v)$ for every edge $u v$ of $G$. A graph $G$ is $w$-coherent $k$-choosable if, given any list assignment $L$ such that $|L(v)| \geq k$ for each vertex $v$ of $G$, there is a $w$-coherent $L$-colouring.

Similarly, a $\mathbb{Z}_{p}$-colouring $c$ of $G$ is $w$-circular if $|c(u)-c(v)|_{p} \geq w(u v)$ for every edge $u v$ of $G$. A graph $G$ is $w$-circular $k$-choosable if, given any $\mathbb{Z}_{p}$-list assignment $L$ such that $|L(v)| \geq k$ for each vertex $v$ of $G$, there is a $w$-circular $L$-colouring. Clearly, every $w$-circular colouring is a $w$-coherent colouring, and every $w$-coherent $k$-colouring induces a $w$-circular $\left(k+\max _{e \in E(G)} w(e)-1\right)$-circular colouring.

The $w$-degree of a vertex $v$ is $d_{w}(v)=\sum_{e \ni v}(2 w(e)-1)$. The maximum $w$-degree of $G$, denoted $\Delta_{w}(G)$, is $\max \left\{d_{w}(v) \mid v \in V(G)\right\}$. Clearly, using the (one-pass) greedy algorithm, every graph is $w$-circular $\left(\Delta_{w}(G)+1\right)$-choosable.

The $w$-semi-degree of a vertex $v$ is $\tilde{d}_{w}(v)=\sum_{e \ni v} w(e)$, and the maximum semi-degree of a graph $G$ is $\tilde{\Delta}_{w}(G)=\max \left\{\tilde{d}_{w}(v) \mid v \in V(G)\right\}$. Using the many-passes greedy algorithm, McDiarmid [14] proved that every graph $G$ is $w$-coherent $\left(\tilde{\Delta}_{w}(G)+1\right)$-choosable. Therefore every graph $G$ is $w$-circular $\left(\tilde{\Delta}_{w}(G)+\max _{e \in E(G)} w(e)\right)$-choosable.

For a digraph $D$, we denote by $E_{D}^{+}(v)$ the set of arcs with $v$ as their tails, and by $E_{D}^{-}(v)$ the set of arcs with $v$ as their heads. Similarly, if $D$ is a digraph, its $w$-outdegree is $d_{w}^{+}(v)=\sum_{e \in E_{D}^{+}(v)}(2 w(e)-1)$.

Extending a result of Norine, Wong and Zhu [17] (Theorem 33), which itself extended a famous result of Alon and Tarsi [3] (Theorem 32), we use the Combinatorial Nullstellensatz to prove that if a graph $G$ admits an orientation $D$ with certain property, then for any $\mathbb{Z}_{p}$-list assignment $L$ such that $|L(v)| \geq$ $d_{w}^{+}(v)+1$ for each vertex $v$, there is a $w$-circular $L$-colouring (Lemma 34). In particular, if $D$ is an orientation of $G$ that contains no odd directed cycles, then it has the required property (Theorem 34 . From this we deduce that if $G$ is bipartite, then it is $w$-circular $(M+1)$-choosable for $M=\sum_{i}(2 i-$ 1) $\left\lceil\operatorname{Mad}\left(G_{i}\right) / 2\right\rceil$, where $G_{i}$ is the graph induced by the edges of weight $i$.

\subsection{Restricted graph classes}

Let $\mathcal{G}$ and $\mathcal{H}$ be two graph classes. For any parameter $A$ among $\mathrm{BBC}_{q}, \mathrm{CBC}_{q}$ and $\mathrm{CBC}_{q}^{\ell}$, we define $A(\mathcal{G}, \mathcal{H})$ as the maximum value of $A(G, H)$ over all pairs $(G, H)$ such that $G \in \mathcal{G}, H \in \mathcal{H}$ and $H$ is a subgraph of $G$. Let $\mathcal{P}, \mathcal{F}$ and $\mathcal{M}$ be the classes of planar graphs, forests, and matchings respectively.

In Section 5, we consider the case when $G$ is in $\mathcal{P}$ and $H$ is in one of the three classes $\mathcal{P}, \mathcal{F}$ and $\mathcal{M}$. Inequality $(1)$ and the Four-Colour Theorem imply $\mathrm{BBC}_{q}(\mathcal{P}, \mathcal{P}) \leq 3 q+1$ and $\mathrm{CBC}_{q}(\mathcal{P}, \mathcal{P}) \leq 4 q$. Moreover, $\mathrm{BBC}_{q}(G, G)=3 q+1$ and $\mathrm{CBC}_{q}(G, G)=4 q$, for any 4-chromatic planar graph $G$. Thus

$$
\mathrm{BBC}_{q}(\mathcal{P}, \mathcal{P})=3 q+1 \text { and } \mathrm{CBC}_{q}(\mathcal{P}, \mathcal{P})=4 q .
$$

Extending the celebrated planar 5-choosability proof due to Thomassen [19], Havet et al. [10] proved the that if $G$ is planar, then $\operatorname{CBC}_{q}^{\ell}(G, G) \leq 8 q-3$. On the other hand, they constructed planar graphs $G$ 
for which $\mathrm{CBC}_{q}^{\ell}(G, G) \geq 6 q-2$. Therefore,

$$
6 q-2 \leq \mathrm{CBC}_{q}^{\ell}(\mathcal{P}, \mathcal{P}) \leq 8 q-3 .
$$

But the exact value of $\mathrm{CBC}_{q}^{\ell}(\mathcal{P}, \mathcal{P})$ is still unknown.

Problem 7 What is the exact value of $\mathrm{CBC}_{q}^{\ell}(\mathcal{P}, \mathcal{P})$ ?

This problem is very closely related to the one posed by Mohar [15].

Problem 8 (Mohar [15]) What is the best upper bound on circular choosability for planar graphs?

Clearly, $\mathrm{BBC}_{q}(\mathcal{P}, \mathcal{M}) \leq \mathrm{BBC}_{q}(\mathcal{P}, \mathcal{F}) \leq \mathrm{BBC}_{q}(\mathcal{P}, \mathcal{P}) \leq 3 q+1$. However, better upper bounds have been obtained for $\mathrm{BBC}_{q}(\mathcal{P}, \mathcal{M})$ and $\mathrm{BBC}_{q}(\mathcal{P}, \mathcal{F})$. Havet et al. [11] proved that for $q \geq 4$, $\mathrm{BBC}_{q}(\mathcal{P}, \mathcal{F})=q+6$. They also proved that $\mathrm{BBC}_{3}(\mathcal{P}, \mathcal{F}) \leq 9$, and conjectured the following.

Conjecture 9 (Havet et al. [11] ) $\mathrm{BBC}_{3}(\mathcal{P}, \mathcal{F})=8$.

Inequality 1 and the Four-Colour Theorem imply that $\mathrm{BBC}_{2}(\mathcal{P}, \mathcal{F}) \leq 7$. Broersma et al. [5] showed $\mathrm{BBC}_{2}(\mathcal{P}, \mathcal{F}) \geq 6$ and conjectured the following.

Conjecture 10 (Broersma et al. [5]) $\mathrm{BBC}_{2}(\mathcal{P}, \mathcal{F})=6$.

Havet et al. [11] showed that Conjecture 10 implies Conjecture 9

They also proved that $\mathrm{CBC}_{q}(\mathcal{P}, \mathcal{F}) \leq 2 q+4$ and conjectured that this upper bound can be reduced by at least one.

Conjecture $11 \mathrm{CBC}_{q}(\mathcal{P}, \mathcal{F}) \leq 2 q+3$.

It might even be possible that the bound $2 q+3$ is not optimal.

Regarding matching backbones, Broersma et al. [6] proved that for $\mathrm{BBC}_{q}(\mathcal{P}, \mathcal{M})=q+3$ for all $q \geq 3$ and conjecture that the same holds for $q=2$.

Conjecture $12 \mathrm{BBC}_{2}(\mathcal{P}, \mathcal{M})=5$.

By Inequality 22, the fact that $\mathrm{BBC}_{q}(\mathcal{P}, \mathcal{M}) \leq q+3$ implies that $\mathrm{CBC}_{q}(\mathcal{P}, \mathcal{M}) \leq 2 q+2$. The missing case $q=2$ of this inequality was proved by Broersma et al. [6].

Proposition 13 (Broersma et al. [6]) $\operatorname{CBC}_{q}(\mathcal{P}, \mathcal{M}) \leq 2 q+2$.

They also show an example for which the bound is attained for $q=2$, so $\mathrm{CBC}_{2}(\mathcal{P}, \mathcal{M})=6$. For larger value of $q$, it is still open if the bound $2 q+2$ is best possible.

Problem 14 Is it true that $\mathrm{CBC}_{q}(\mathcal{P}, \mathcal{M}) \leq 2 q+1$, for all $q \geq 3$ ?

We also believe that Conjecture 11 and Proposition 13 extend to list circular backbone colouring.

\section{Conjecture $15 \quad$ (i) $\operatorname{CBC}_{q}^{\ell}(\mathcal{P}, \mathcal{F}) \leq 2 q+3$;}

(ii) $\mathrm{CBC}_{q}^{\ell}(\mathcal{P}, \mathcal{M}) \leq 2 q+2$.

In support of both parts of this conjecture, we derive from our result of Section 4 that if $G$ is a bipartite planar graph and $F$ a forest in $G$, then $\operatorname{CBC}_{q}^{\ell}(G, F) \leq 2 q+2$. Observe that by 3 , we have $\mathrm{CBC}_{q}(G, F) \leq 2 q$. We also show that $\mathrm{CBC}_{q}^{\ell}(\mathcal{P}, \mathcal{M}) \leq 2 q+3$ (Theorem 42. Our proof also uses the approach of Thomassen's 5 -choosability proof [19]. 


\section{Bounding $\mathrm{CBC}_{q}(G, H)$ and $\mathrm{CBC}_{q}^{\ell}(G, H)$ in terms of $\chi^{\prime}(H)$ and $\chi^{\ell}(G)$}

Theorem 16 Let $M_{q}(k)=\frac{4\left(k^{2}+1\right)^{2}(2 q-1)}{\log _{2} e^{2}} \cdot 2^{2 k}+1$. Then $\operatorname{CBC}_{q}^{\ell}(G, H) \leq M_{q}\left(\chi^{\ell}(G)\right)$ for any subgraph $H$ of $G$.

Proof: Because $\mathrm{CBC}_{q}^{\ell}(G, H) \leq \mathrm{CBC}_{q}^{\ell}(G, G)$ for any graph $G$, it is sufficient to prove the inequality for $\mathrm{CBC}_{q}^{\ell}(G, G)$. Let $G$ be a graph such that $\chi^{\ell}(G)=k$ and $\mathrm{CBC}_{q}^{\ell}(G, G)>M_{q}(k)$. Let $H$ be the smallest subgraph of $G$ such that $\operatorname{CBC}_{q}^{\ell}(H, H)>M_{q}(k)$. Then $\delta(H)>\frac{M_{q}(k)-1}{2 q-1}=\frac{4\left(k^{2}+1\right)^{2}}{\log _{2} e^{2}} \cdot 2^{2 k}$. But Alon [1] proved that if $\delta(G)>\frac{4\left(k^{2}+1\right)^{2}}{\log _{2} e^{2}} \cdot 2^{2 k}$, then $\chi^{\ell}(G)>k$. Therefore $\chi^{\ell}(H)>k$ and so $\chi^{\ell}(G)>k$, a contradiction.

We believe the bound $M_{q}(k)$ of Theorem 16 is far from being tight. In particular, when $H$ is a matching, we can get an upper bound that is a lot smaller.

Theorem 17 Let $G$ be a graph and $M$ a matching in $G$. Then

(i) $\mathrm{CBC}_{2}^{\ell}(G, M) \leq 2 \cdot \chi^{\ell}(G)+1$,

(ii) for any $q \geq 3$ and any $\alpha>1$, there is $\beta_{q}=\beta_{q}(\alpha)$ such that $\operatorname{CBC}_{q}^{\ell}(G, M) \leq 2 \alpha \cdot \chi^{\ell}(G)+\beta_{q}$.

In order to prove this theorem, we need some definitions. Let $A$ and $B$ be two subsets of $\mathbb{N}$ (resp. $\mathbb{Z}_{p}$ ). We say that $A$ and $B$-interfere if there exists $a \in A$ and $b \in B$ such that $a \neq b$ and $|a-b|<q$ (resp. $\left.|a-b|_{p}<q\right)$.

For any pair of non-negative integers, we define $f_{q}(a, b)$ to be the smallest integer $m$ such that for any two subsets $A$ and $B$ of $\mathbb{N}$ of cardinality at least $m$, there exist two non- $q$-interfering sets $A^{\prime} \subset A$ and $B^{\prime} \subset B$ such that $\left|A^{\prime}\right|=a,\left|B^{\prime}\right|=b$. Similarly, let $g_{q}(a, b)$ be the smallest integer $m$ such that for any $p$ and any two subsets $A$ and $B$ of $\mathbb{Z}_{p}$ of cardinality at least $m$, there exist two non- $q$-interfering sets $A^{\prime} \subset A$ and $B^{\prime} \subset B$ such that $\left|A^{\prime}\right|=a,\left|B^{\prime}\right|=b$. These two functions are closely related.

\section{Proposition 18}

$$
f_{q}(a, b) \leq g_{q}(a, b) \leq f_{q}(a, b)+q-1 .
$$

Proof: If two sets $q$-interfere in $\mathbb{N}$, then they also $q$-interfere in $\mathbb{Z}_{r}$ (for $r$ sufficiently large). So $f_{q}(a, b) \leq$ $g_{q}(a, b)$. Now let $C$ and $D$ be two sets in $\mathbb{Z}_{p}$ of cardinality $f_{q}(a, b)+q-1$, and consider $A=C \backslash$ $\{0, \ldots, q-2\}$ and $B=D \backslash\{0, \ldots, q-2\}$. Then $|A|,|B| \geq f_{q}(a, b)$, so there exist two sets $A^{\prime} \subset A$ and $B^{\prime} \subset B$ of cardinality $a$ and $b$ respectively which do not $q$-interfere in $\mathbb{N}$. But by the definitions of $A$ and $B, A^{\prime} \subset C, B^{\prime} \subset D$ and $A^{\prime}$ and $B^{\prime}$ cannot $q$-interfere in $\mathbb{Z}_{p}$.

Let us define $h_{q}$ by $h_{q}(k)=g_{q}(k, k)$. The function $h_{q}$ is useful for our purpose as shown by the following lemma.

Lemma 19 Let $G$ be a graph and $M$ a matching in $G$. Then

$$
\mathrm{CBC}_{q}^{\ell}(G, M) \leq h_{q}\left(\chi^{\ell}(G)\right) .
$$


Proof: Let $k=\chi^{\ell}(G)$. Let $L$ be an $h_{q}(k)$-list assignment in $Z_{p}$. By definition of $h_{q}$, for every edge $u v \in M$, we can find two $k$-sets $L^{\prime}(u) \subset L(u)$ and $L^{\prime}(v) \subset L(v)$ that do not $q$-interfere. Since $G$ is $k$-choosable, there is an $L^{\prime}$-colouring $c$ of $G$.

We now show that $c$ is a $q$-backbone colouring of $(G, M)$. First, for any edge $x y \in E(G), c(x) \neq c(y)$. For any edge $u v \in M, c(u) \neq c(v)$. In addition, since $L^{\prime}(u)$ and $L^{\prime}(v)$ do not $q$-interfere, $|c(u)-c(v)|_{p} \geq$ $q$.

Notation For two integers $a$ and $b$, we denote by $[a, b]^{p}$, the set $\{a, a+1, \ldots, b\}$, where the numbers are modulo $p$. For every integer $a$, we denote by $[a]_{q}^{p}$ the set $[a-q+1, a+q-1]^{p}$. Very often $p$ is either clear from the context or implicit and we omit the superscript $p$.

For a real number $\alpha$ and a positive integer $q$, we define the sequence $\psi_{q, \alpha}(i)$ by $\psi_{q, \alpha}(0)=1$ and $\psi_{q, \alpha}(i+1)=\left\lfloor\alpha \cdot \psi_{q, \alpha}(i)+1\right\rfloor$. Observe that $\psi_{q, \alpha}(i) \geq \alpha^{i}$. Thus if $\alpha>1$, then $\psi_{q, \alpha}(i)-(q+1) i$ tends to infinity when $i$ tends to infinity. Let $i^{*}=i^{*}(q, \alpha)$ be the smallest positive integer such that $(q-1) i^{*}<\psi_{q, \alpha}\left(i^{*}\right)$ and set $R(q, \alpha)=(2 q-3) \psi_{q, \alpha}\left(i^{*}\right)-1$.

Lemma $20 \quad$ (i) $f_{2}(a, b) \leq a+b$.

(ii) For any integer $q \geq 3$ and any real number $\alpha \geq 1$, we have $f_{q}(a, b) \leq \alpha(a+b)+R(q, \alpha)$.

Proof: (i) We prove the result by induction on $a+b$, the result holding trivially when $a=0$ or $b=0$. Let $A$ and $B$ be two sets of integers of size $a+b$, and let $x$ be the minimum of $A \cup B$.

Suppose first $x \in A \cap B$. Let $C=A \backslash\{x, x+1\}$, and $D=B \backslash\{x, x+1\}$. Then $|C|,|D| \geq a+b-2$, so by the induction hypothesis, there exist two non-2-interfering sets $C^{\prime} \subset C$ and $D^{\prime} \subset D$ such that $\left|C^{\prime}\right|=a-1,\left|D^{\prime}\right|=b-1$. Setting $A^{\prime}=C^{\prime} \cup\{x\}$ and $B^{\prime}=D^{\prime} \cup\{x\}$, we obtain the desired sets.

Suppose now $x \notin A \cap B$. Then $x$ is only in one of these two sets, say $A$. Let $C=A \backslash\{x\}$, and $D=B \backslash\{x+1\}$. Then $|C|,|D| \geq a+b-1$, so by the induction hypothesis, there exist two non-2interfering sets $C^{\prime} \subset C$ and $D^{\prime} \subset D$ such that $\left|C^{\prime}\right|=a-1,\left|D^{\prime}\right|=b$. Setting $A^{\prime}=C^{\prime} \cup\{x\}$ and $B^{\prime}=D^{\prime}$, we obtain the desired sets.

(ii) We prove the result by induction on $a+b$. Let $A$ and $B$ be two sets of integers of size $\alpha(a+b)+$ $R(q, \alpha)$.

Assume $a<\psi_{q, \alpha}\left(i^{*}\right)$. Then one can choose any $a$-subset $A^{\prime}$ of $A$. It forbids at most $a \times(2 q-2)$ elements to be chosen in $B$. So since $B$ is of cardinality at least $b+a+R(q, \alpha) \geq b+(2 q-2) a$, we can find a $b$-subset $B^{\prime}$ that does not $q$-interfere with $A^{\prime}$.

We get the result similarly if $b<\psi_{q, \alpha}\left(i^{*}\right)$. Henceforth we now assume that $a$ and $b$ are both greater or equal to $\psi_{q, \alpha}\left(i^{*}\right)$.

Let $x$ be the smallest element of $A \cup B$. Without loss of generality, we may assume that $x \in A$. Consider $C_{1}=A \backslash\{x\}$ and $D_{1}=B \backslash[x, x+q-1]$. If $|B \cap[x, x+q-1]| \leq \alpha \cdot \psi_{q, \alpha}(0)$, then $C_{1}$ and $D_{1}$ are both of cardinality at least $\alpha(a+b-1)+R(q, \alpha)$. Hence by the induction hypothesis, we can find an $(a-1)$-set $C^{\prime}$ in $C_{1}$ and a $b$-set $B^{\prime}$ in $D_{1}$ which do not $q$-interfere. Then, $A^{\prime}=C^{\prime} \cup\{x\}$ and $B^{\prime}$ are the desired sets. Hence we may assume that at least $\psi_{q, \alpha}(1)$ elements of $B$ are in $[x, x+q-1]$.

We now extend the argument by removing the $\psi_{q, \alpha}(1)$ smallest elements of $B$. For any $k$, let $A(k)$ (resp. $B(k)$ ) be the set of the $k$ smallest elements in $A$ (resp. $B$ ). Consider $D_{2}=B \backslash B\left(\psi_{q, \alpha}(1)\right)$ and $C_{2}=A \backslash[x, x+2(q-1)]$. If $|A \cap[x, x+2(q-1)]| \leq \alpha \cdot \psi_{q, \alpha}(1)$, then $C_{2}$ and $D_{2}$ are both of cardinality at least $\alpha\left(a+b-\psi_{q, \alpha}(1)\right)+R(q)$. Hence by the induction hypothesis, we can find an $a$-set $A^{\prime}$ in $C_{2}$ and a 
$\left(b-\psi_{q, \alpha}(1)\right)$-set $D^{\prime}$ in $D_{2}$ which do not $q$-interfere. Then, $A^{\prime}$ and $B^{\prime}=D^{\prime} \cup B\left(\psi_{q, \alpha}(1)\right)$ are the desired sets. (They do not interfere because, as we proved in the last paragraph, $B\left(\psi_{q, \alpha}(1)\right) \subseteq[x, x+q-1]$ ). Hence we may assume that $A\left(\psi_{q, \alpha}(2)\right) \subseteq[x, x+2(q-1)]$.

We continue to extend the argument for $\psi_{q, \alpha}(i)$, with $2 \leq i \leq i^{*}$, alternating the roles of $A$ and $B$, as follows. If $i$ is odd, consider $C_{i}=A \backslash A\left(\psi_{q, \alpha}(i-1)\right)$ and $D_{i}=B \backslash[x, x+i(q-1)]$. If $\mid B \cap[x, x+i(q-$ $1)] \mid \leq \alpha \cdot \psi_{q, \alpha}(i-1)$, then $C_{i}$ and $D_{i}$ are both of cardinality at least $\alpha\left(a+b-\psi_{q, \alpha}(i-1)\right)+R(q, \alpha)$. Hence by the induction hypothesis, we can find an $\left(a-\psi_{q, \alpha}(i-1)\right)$-set $C^{\prime}$ in $C_{i}$ and a $b$-set $B^{\prime}$ in $D_{i}$ which do not $q$-interfere. Then, $A^{\prime}=C^{\prime} \cup A\left(\psi_{q, \alpha}(i-1)\right)$ and $B^{\prime}$ are the desired sets. Hence we may assume that $B\left(\psi_{q, \alpha}(i)\right) \subset[x, x+i(q-1)]$. If $i$ is even, consider $D_{i}=B \backslash B\left(\psi_{q, \alpha}(i-1)\right)$ and $C_{i}=A \backslash[x, x+i(q-1)]$. If $|A \cap[x, x+i(q-1)]| \leq \alpha \cdot \psi_{q, \alpha}(i-1)$, then $C_{i}$ and $D_{i}$ are both of cardinality at least $\alpha\left(a+b-\psi_{q, \alpha}(i-1)\right)+R(q, \alpha)$. Hence by the induction hypothesis, we can find an $a$-set $A^{\prime}$ in $C_{i}$ and a $\left(b-\psi_{q, \alpha}(i-1)\right)$-set $B^{\prime}$ in $D_{i}$ which do not $q$-interfere. Then, $A^{\prime}$ and $B^{\prime}=D^{\prime} \cup B\left(\psi_{q, \alpha}(i)\right)$ are the desired sets. Hence we may assume that $A\left(\psi_{q, \alpha}(i)\right) \subset[x, x+i(q-1)]$.

So, depending on the parity of $i^{*},\left[x, x+i^{*}(q-1)\right]$ contains at least $\psi_{q, \alpha}\left(i^{*}\right)$ vertices of either $A$ or $B$. But this is impossible, because $\left[x, x+i^{*}(q-1)\right]$ has cardinality less than $\psi_{q, \alpha}(i-1)$ by definition of $i^{*}$.

\section{Corollary $21 \quad$ (i) $g_{2}(a, b) \leq a+b+1$.}

(ii) for any $q \geq 3$ and any $\alpha>1, g_{q}(a, b) \leq \alpha(a+b)+R(q, \alpha)+q-1$.

Remark $22 a+b+1$ may not be replaced by $a+b$, even if $a=b$, in Corollary 21 (i). Just consider $a=b$ and $\mathbb{Z}_{4 a}$ with $A$ the set of even numbers and $B$ the set of odd numbers. One can check that any pair of two $a$-sets $A^{\prime} \subset A$ and $B^{\prime} \subset B$ 2-interfere.

Theorem 17 derives directly from Corollary 21 and Lemma 19 . It can also be generalized to any subgraph $H$. Indeed in the very same way as Lemma 19 , we can prove the following.

Lemma 23 Let $G$ be a graph, $H$ a subgraph of $G$ and $M$ a matching in $H$. Then

$$
\operatorname{CBC}_{q}^{\ell}(G, H) \leq h_{q}\left(\operatorname{CBC}_{q}^{\ell}(G, H-M)\right) .
$$

Hence by induction, we get the following theorem, where $\chi^{\prime}(H)$ denotes the chromatic index of $H$.

Theorem 24 Let $G$ be a graph and $H$ a subgraph of $G$ and let $q \geq 2$ be an integer. Then $\operatorname{CBC}_{q}^{\ell}(G, H) \leq$ $2^{\chi^{\prime}(H)}(1+o(1)) \chi^{\ell}(G)$.

Remark 25 In the case $q=2$, one can get rid of the $o(1)$ term and show that $\operatorname{CBC}_{2}^{\ell}(G, H) \leq 2^{\chi^{\prime}(H)} \chi^{\ell}(G)+$ 1. It suffices to observe that we can remove one fixed colour so that the colours behave like on $\mathbb{N}$. Then for each matching $M$ of a proper edge-colouring, we must divide the list by 2 so that there is no 2 -interference between vertices linked by an edge in $M$.

\section{2-choosable graphs}

In this section we study list backbone colouring of 2-choosable graphs.

Let $t$ and $q$ be two positive integers. The $(t, q)$-kernel of $(G, H)$ is the maximal subset $K$ of $V(G)$ such that $d_{G[K]}(v)+(2 q-2) d_{H[K]}(v) \geq t$ for every vertex $v \in K$. Observe that if $t \geq 2 q$, then the graph $G[K]$ is a subgraph of the heart of $G$.

By convention, $\operatorname{CBC}_{q}^{\ell}(N, N)=1$ if $N$ is the null graph. 
Lemma 26 Let $t$ and $q$ be two positive integers, and let $G$ be a graph and $M$ a matching in $G$, and let $K$ be its $(t, q)$-kernel. Then $\mathrm{CBC}_{q}^{\ell}(G, M) \leq t$ if and only if $\mathrm{CBC}_{q}^{\ell}(G[K], M[K]) \leq t$.

Proof: Clearly, $\mathrm{CBC}_{q}^{\ell}(G[K], M[K]) \leq \mathrm{CBC}_{q}^{\ell}(G, M)$, so we only need to prove that $\mathrm{CBC}_{q}^{\ell}(G, M) \leq t$ if $\mathrm{CBC}_{q}^{\ell}(G[K], M[K]) \leq t$.

Let $L$ be an $t$-list assignment on $G$ in $Z_{r}$. It is easy to see that for any subgraph $G^{\prime}$ of $G$, if there is a vertex $v$ such that $d_{G^{\prime}}(v)+(2 q-2) d_{H^{\prime}}(v)<t$, where $H^{\prime}=H\left[V\left(G^{\prime}\right)\right]$, then any $q$-backbone $L$-colouring of $\left(G^{\prime}-v, H^{\prime}-v\right)$ can be extended into a $q$-backbone $L$-colouring of $\left(G^{\prime}, H^{\prime}\right)$.

Now by definition of $K$, there exists a sequence $v_{1}, \ldots, v_{p}$ of $V(G) \backslash K$ such that for all $1 \leq i \leq p$, $d_{G_{i}}(v)+(2 q-2) d_{H_{i}}(v)<t$, where $G_{i}=G\left[K \cup\left\{v_{1}, \ldots, v_{i}\right\}\right]$ and $H_{i}=H\left[K \cup\left\{v_{1}, \ldots, v_{i}\right\}\right]$. Therefore any $q$-backbone $L$-colouring of $(G[K], M[K])$ can be iteratively extended into a $q$-backbone $L$-colouring of $\left(G_{1}, H_{1}\right),\left(G_{2}, H_{2}\right), \ldots\left(G_{p}, H_{p}\right)=(G, H)$.

Lemma 26 and Theorem 6immediately imply the following.

Corollary 27 Let $G$ be a graph and $H$ a nonempty subgraph of $G$. If the heart of $G$ is a single vertex or an even cycle, then $\mathrm{CBC}_{q}^{\ell}(G, H)=2 q$.

Theorem 28 If $G$ is a 2-choosable graph and $M$ a matching, then $\mathrm{CBC}_{q}^{\ell}(G, M)=2 q$.

Proof: Clearly, it is enough to prove the statement for connected graphs. So we may assume that $G$ is connected.

Let $H$ be the heart of $G$, and let $N=M[V(H)]$. Observe that if $H$ is a $\theta_{2 m, 2,2}$ for some integer $m \geq 1$, with $x$ and $y$ its two vertices of degree three, then $H-\{x, y\}$ has three connected components of odd order. Hence, there is a vertex $v$ of $H \backslash\{x, y\}$ that is not matched in $N$, and for which $d_{H}(v)+(2 q-2) d_{N}(v)=$ $d_{H}(v)<2 q$.

By Theorem 4 it follows that the $(2 q, q)$-kernel of $(G, M)$ is either the empty set, or a set $K$ such that $G[K]$ is an even cycle and $M[K]$ a perfect matching of $G[K]$. Hence, by Theorem $6 . \mathrm{CBC}_{q}^{\ell}(G[K], M[K]) \leq$ $2 q$, and so by Lemma 26 . $\operatorname{CBC}_{q}^{\ell}(G, M) \leq 2 q$.

Remark 29 In the above proof, Theorem 6 is not necessarily required as it is easy to see that $\mathrm{CBC}_{q}^{\ell}(G, M) \leq$ $2 q$ for an even cycle $G$ and a matching $M$.

Theorem 30 If $G$ is a 2-choosable graph and $H$ is a subgraph of $G$ not containing its heart, then $\mathrm{CBC}_{q}^{\ell}(G, H) \leq 2 q+1$.

Proof: Again it is enough to prove the statement for $G$ connected. If $H$ does not contain the heart of $G$, then, using Theorem 4, one easily sees that the $(2 q+1, q)$-kernel of $(G, H)$ is either the empty graph or an even cycle. Therefore Theorem 6 and Lemma 26 yield the result. 


\section{Weighted circular list colouring via the Combinatorial Nullstel- lensatz}

In this section we shall use the following theorem, called the Combinatorial Nullstellensatz, to establish some results on circular backbone colouring.

Theorem 31 Let $F$ be an arbitrary field, and let $P=P\left(x_{1}, \ldots, x_{n}\right)$ be a polynomial in $F\left[x_{1}, \ldots, x_{n}\right]$. Suppose that the degree $\operatorname{deg}(P)$ of $P$ is $\sum_{i=1}^{n} t_{i}$, where each $t_{i}$ is a non-negative integer, and suppose the coefficient of $\prod_{i=1}^{n} x_{i}^{t_{i}}$ in $P$ is non-zero. Then, if $S_{1}, \ldots, S_{n}$ are subsets of $F$ with $\left|S_{i}\right|>t_{i}$, there are $s_{1} \in S_{1}, \ldots, s_{n} \in S_{n}$ so that

$$
P\left(s_{1}, \ldots, s_{n}\right) \neq 0
$$

Let $G$ be a graph with vertex set $\left\{v_{1}, \ldots, v_{n}\right\}$. The graph polynomial $f_{G}$ of $G$ is defined by $f_{G}\left(x_{1}, \ldots, x_{n}\right)=$ $\prod\left\{\left(x_{i}-x_{j}\right) \mid i<j, v_{i} v_{j} \in E(G)\right\}$. Alon and Tarsi [3] applied the Combinatorial Nullstellensatz to the graph polynomial to obtain the following theorem on choosability.

Theorem 32 (Alon and Tarsi [3]) Let D be an orientation of a graph $G$, and let ee $(D)$ and oe $(D)$ respectively the numbers of even and odd eulerian spanning subdigraphs of $D$. If ee $(D) \neq$ oe $(D)$, then for any list assignment $L$ such that $|L(v)| \geq d^{+}(v)+1$ for all $v$, the graph $G$ is $L$-choosable.

Let $G$ be a graph with vertex set $\left\{v_{1}, v_{2}, \ldots, v_{n}\right\}$ and let $p$ and $q$ be two positives integers. Its $(p, q)$ circular polynomial of $G$ is

$$
C P_{p, q}^{G}\left(x_{1}, x_{2}, \ldots, x_{n}\right)=\prod_{\substack{v_{j} \\ v_{j^{\prime}} \in E(G) \\ j<j^{\prime}}} \prod_{\substack{k=-q+1 \\ j^{\prime}}}^{q-1}\left(x_{j}-\exp (2 \pi i k / p) x_{j^{\prime}}\right)
$$

Let $\gamma:\{0, \ldots, p-1\} \rightarrow \mathbb{C}$ be the function defined by $\gamma(k)=\exp (2 \pi i k / p)$. It is obvious that a mapping $c: V(G) \rightarrow\{0, \ldots, p-1\}$ is a $(p, q)$-colouring of $G$ if and only if

$$
C P_{p, q}^{G}\left(\gamma\left(c\left(v_{1}\right)\right), \gamma\left(c\left(v_{2}\right)\right), \ldots, \gamma\left(c\left(v_{n}\right)\right) \neq 0 .\right.
$$

Applying the Combinatorial Nullstellensatz to the $(p, q)$-circular polynomials, Norine, Wong and Zhu [17] established a generalization of Theorem 32 , from which they deduced the following.

Theorem 33 (Norine, Wong and Zhu [17]) Suppose $G$ is a graph and D is an orientation of $G$ which contains no odd directed cycles. Let $L$ be a $\mathbb{Z}_{p}$-list assignment for $G$ such that $|L(v)|=(2 q-1) d_{D}^{+}(v)+1$ for each vertex $v$. Then $G$ is $L$ - $(p, q)$-colourable.

In this section, we shall extend Theorem 33 to $w$-circular colouring. We will prove the following theorem.

Theorem 34 Suppose $G$ is a graph with a positive integer edge weighting $w$. Suppose moreover that $D$ is an orientation of $G$ which contains no odd directed cycles. If $L$ is a $\mathbb{Z}_{p}$-list assignment for $G$ such that $|L(v)|=d_{w}^{+}(v)+1$ for each vertex $v$, then $G$ is $w$-circularly L-colourable. 
Let $G$ be a graph with vertex set $\left\{v_{1}, v_{2}, \ldots, v_{n}\right\}, w$ an edge weight of $G$, and $p$ a positive integer. The $(p, w)$-circular polynomial of $G$ is

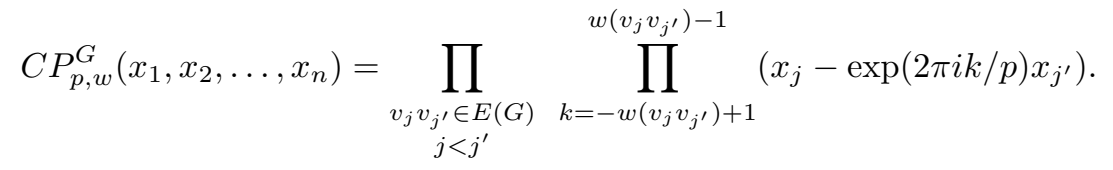

Observe that when $w(e)=q$ for all $e \in E(G)$, then $C P_{p, w}^{G}=C P_{p, q}^{G}$. Let $\gamma: \mathbb{Z}_{p} \rightarrow \mathbb{C}$ be defined as $\gamma(l)=\exp (2 \pi i l / p)$ for $l \in \mathbb{Z}_{p}$. It is obvious that a mapping $c: V \rightarrow \mathbb{Z}_{p}$ is a $w$-circular colouring of $G$ if and only if

$$
C P_{p, w}^{G}\left(\gamma\left(c\left(v_{1}\right)\right), \gamma\left(c\left(v_{2}\right)\right), \ldots, \gamma\left(c\left(v_{n}\right)\right)\right) \neq 0 .
$$

Thus, the graph $G$ is $w$-circularly $L$-colourable if and only if there exist $s_{1} \in L\left(v_{1}\right), s_{2} \in L\left(v_{2}\right), \ldots, s_{n} \in$ $L\left(v_{n}\right)$ such that $C P_{p, w}^{G}\left(\gamma\left(s_{1}\right), \gamma\left(s_{2}\right), \ldots, \gamma\left(s_{n}\right)\right) \neq 0$.

The degree of $C P_{p, w}^{G}$ is $\sum_{e \in E}(2 w(e)-1)=\sum_{j=1}^{n} d_{w}^{+}\left(v_{j}\right)$. Thus by Theorem 31 if there is an orientation $D$ such that the coefficient of the monomial $\prod_{j=1}^{n} x_{j}^{d_{w}^{+}\left(v_{j}\right)}$ is nonzero, the graph $G$ is $w$ circularly $L$-colourable for any $\mathbb{Z}_{p}$-list assignment $L$ such that $|L(v)|=d_{w}^{+}(v)+1$ for all $v \in V(G)$.

Let $D$ be an orientation of $G$. A non-negative integer mapping $\phi: E(D) \rightarrow \mathbb{Z}$ is w-consistent if $0 \leq \phi(e) \leq 2 w(e)-1$ for all $e \in E(D)$. It is eulerian if for every vertex $v$,

$$
\sum_{e \in E_{D}^{+}(v)} \phi(e)=\sum_{e \in E_{D}^{-}(v)} \phi(e)
$$

A mapping that is consistent and eulerian is said to be nice. A mapping $\phi$ is called even (respectively, $o d d)$ if $\sum_{e \in E(D)} \phi(e)$ is even (respectively, odd).

For an $\operatorname{arc}\left(v_{j}, v_{j^{\prime}}\right)$ of $D$ of weight $q$ and for $0 \leq \phi(e) \leq 2 q-1$, the coefficient of $x_{j}^{2 q-1-\phi(e)} x_{j^{\prime}}^{\phi(e)}$ in $\prod_{k=-q+1}^{q-1}\left(x_{j}-\exp (2 \pi i k / p) x_{j^{\prime}}\right)$ is equal to

$$
\sum_{\substack{J \subseteq\{-q+1, \ldots, q-1\} \\|J|=\phi(e)}} \prod_{j \in J}(-\exp (2 \pi i j / p))=(-1)^{\phi(e)} a_{\phi(e)}(p, q),
$$

where

$$
a_{\phi(e)}(p, q)=\sum_{\substack{J \subseteq\{-q+1, \ldots, q-1\} \\|J|=\phi(e)}} \prod_{j \in J} \exp (2 \pi i j / p)
$$

It is easy to see that a $w$-consistent mapping $\phi$ makes a contribution to the monomial $\prod_{j=1}^{n} x_{j}^{d_{w}^{+}\left(v_{j}\right)}$ if and only if it is nice. Moreover, such a mapping contributes for an amount

$$
A(p, w, \phi)=\prod_{e \in E(D)}(-1)^{\phi(e)} a_{\phi(e)}(p, w(e)) .
$$


Therefore, the coefficient of $\prod_{j=1}^{n} x_{j}^{d_{w}^{+}\left(v_{j}\right)}$ in $C P_{p, w}^{G}$ is

$$
\sum_{\phi \text { nice }} \prod_{e \in E(D)}(-1)^{\phi(e)} a_{\phi(e)}(p, w(e))=\sum_{\phi \text { nice and even }} A(p, w, \phi)-\sum_{\phi \text { nice and odd }} A(p, w, \phi) .
$$

Hence, we get the following lemma.

Lemma 35 Suppose a graph $G$ has an orientation D for which

$$
\sum_{\phi \text { nice and even }} A(p, w, \phi) \neq \sum_{\phi \text { nice and odd }} A(p, w, \phi) .
$$

If $L$ is a $\mathbb{Z}_{p}$-list assignment for $G$ such that $|L(v)|=d_{w}^{+}(v)+1$ for each vertex $v$, then $G$ is $w$-circularly L-colourable.

Observe now that each $a_{l}(p, q)$ is a real because it is equal to its conjugate. We can also see that $a_{0}(p, q)=a_{2 q-1}(p, q)=1$. A result of Evans and Montgomery [8] implies that if $p \geq 2 q$, then the values of $a_{l}(p, q)$, for fixed $p$ and $q$, are unimodal. It follows that all values of $a_{l}(p, q)$ are at least 1 - Norine, Wong and Zhu proved this from first principles for certain values of $p$ and $q$ [17].

Lemma 36 Let $p$ and $q$ be two positive integers such that $p \geq 2 q$. For any $0 \leq l \leq 2 q-1, a_{l}(p, q) \geq 1$.

From Lemmas 35 and 36 , we now derive Theorem 34

Proof Proof of Theorem 34; By Lemma 35, it suffices to show that

$$
\sum_{\phi \text { nice and even }} A(p, w, \phi) \neq \sum_{\phi \text { nice and odd }} A(p, w, \phi) .
$$

For a nice mapping $\phi$, let $D_{\phi}$ be the multi-digraph obtained from $D$ by replacing each $\operatorname{arc}\left(v_{j}, v_{j^{\prime}}\right)$ by $\phi(e)$ parallel arcs from $v_{j}$ to $v_{j^{\prime}}$. Since $\phi$ is eulerian, then $D_{\phi}$ is an eulerian multi-digraph. Each directed cycle of $D_{\phi}$ corresponds to a directed cycle of $D$. Since $D$ has no directed cycle of odd length, $D_{\phi}$ has no directed cycle of odd length. Thus $\left|E\left(D_{\phi}\right)\right|$ is even, i.e. $\sum_{e \in E(D)} \phi(e)$ is even. So $D$ has no odd nice mapping, and so $\sum_{\phi}$ nice and odd $A(p, w, \phi)=0$.

Now Lemma 36 implies that all the $A(p, w, \phi)$ are positive. But there is at least one nice even mapping, namely the one for which $\phi(e)=0$ for all $e \in E(D)$. So $\sum_{\phi}$ nice and even $A(p, w, \phi)>0$.

We shall now apply Theorem 34 . We need the following simple lemma which was first proved by Hakimi [9]. It also appears independently in several papers [18, 2, 3].

Lemma 37 A graph $G$ has an orientation $D$ with maximum outdegree at most $\Delta^{+}$if and only if $\operatorname{Mad}(G) / 2 \leq$ $\Delta^{+}$.

For a graph $G$ and an edge weighting $w$, we define $M(G, w):=\sum_{i \in \mathbb{N}^{*}}(2 i-1)\left\lceil\operatorname{Mad}\left(G_{i}\right) / 2\right\rceil$, where $G_{i}$ is the subgraph of $G$ induced by the edges of weight $i$, for all $i \in \mathbb{N}^{*}$.

Corollary 38 Let $G$ be a graph and $w$ an edge-weighting. Then $G$ has an orientation $D$ such that $d_{w}^{+}(v) \leq M(G, w)$ for all $v \in V$. 
Proof: It suffices to orient each $G_{i}$ separately in such a way that the maximum outdegree is at most $\left\lceil\operatorname{Mad}\left(G_{i}\right) / 2\right\rceil$, which is possible by Lemma 37 The union of these orientations yields the desired one.

Theorem 34 and Corollary 38 immediately yield the following.

Theorem 39 Let $G$ be a bipartite graph and $w$ an edge weighting. Then $G$ is $w$-circular $(M(G, w)+1)$ choosable.

Proof: By Corollary $38, G$ admits an orientation $D$ such that $d_{w}^{+}(v) \leq M(G, w)$ for all $v \in V$. Since $G$ is bipartite, it has no odd cycles and so $D$ has no directed odd cycles. Hence by Theorem $34, G$ is $(M(G, w)+1)$-choosable.

\section{A stronger result for planar graphs}

If $G$ is a bipartite planar graph then $\operatorname{Mad}(G)<4$, and if $T$ is a tree then $\operatorname{Mad}(T)<2$. Thus, Theorem 39 directly implies the following.

Corollary 40 If $G$ is a bipartite planar graph and $T$ a spanning tree of $G$, then $\mathrm{CBC}_{q}^{\ell}(G, T) \leq 2 q+2$.

When the planar graph $G$ is no longer required to be bipartite, we can use an acyclic orientation $D$ with maximum outdegree 5 . Such an orientation exists because a planar graph is 5-degenerate. Considering such an orientation, we derive the following, which corresponds to greedy colouring according to a linear ordering extending $D$ (i.e. an ordering $<\operatorname{such}$ that $x<y$ whenever $(y, x)$ is an arc in $D$ ).

Corollary 41 Let $G$ be a planar graph and $H$ be a subgraph in $G$. Then $\operatorname{CBC}_{q}^{\ell}(G, H) \leq(2 q-2) \Delta(H)+$ 6.

Proof: Let $w$ be the edge-weight defined by $w(e)=q$ if $e \in E(H)$ and $w(e)=1$ if $e \in E(G) \backslash E(H)$. Then for the orientation $D$ with maximum outdegree 5 , we have $\Delta_{w}^{+}(D) \leq(2 q-2) \Delta(H)+5$. Since $D$ is acyclic, it contains no odd directed cycles, and so by Theorem $34,(G, w)$ is circular $((2 q-2) \Delta(H)+6)$ choosable.

In particular, if $G$ is a planar graph and $M$ a matching, $\operatorname{CBC}_{q}^{\ell}(G, M) \leq 2 q+4$. Using a different technique, we now prove a slightly stronger bound.

Theorem 42 Let $G$ be a planar graph and $M$ be a matching in $G$. Then $\operatorname{CBC}_{q}^{\ell}(G, M) \leq 2 q+3$.

We borrow the approach of Thomassen's 5-choosability proof [19], and actually establish a slightly stronger result.

Theorem 43 Let $p$ and $q$ be two positive integers such that $p \geq 2 q+3$. Let $G$ be a near triangulation with outer cycle $C, M$ a matching in $G$ and let $L$ be a list assignment such that:

- $|L(v)| \geq 3$ for all $v \in V(C) \backslash V(M)$;

- $|L(v)| \geq 2 q+1$ for all $v \in V(C) \cap V(M)$;

- $|L(v)| \geq 2 q+3$ otherwise.

Then any valid precolouring of two adjacent vertices of $C$ can be extended to a circular q-backbone $L$-colouring of $(G, M)$. 
To prove this theorem, we need the following lemma.

Lemma 44 Let $q$ be a positive integer and let $S$ and $T$ be two sets of size $2 q$ and $2 q+3$ respectively in $\mathbb{Z}_{p}$. Then there exist two elements $a$ and $b$ of $S$ such that $\left|T \backslash\left([a]_{q} \cup[b]_{q}\right)\right| \geq 3$.

Proof: The result holds trivially when $q=1$.

Let us now prove the case $q=2$. If two elements $a$ and $b$ of $S$ are consecutive in $\mathbb{Z}_{p}$, then $[a]_{2} \cup[b]_{2}=4$, and we trivially have the result. So we may assume that the elements of $S$ are pairwise non-consecutive. Let $1 \leq s_{1}<s_{2}<s_{3}<s_{4} \leq p$ be the elements of $S$ and set $I=\left[s_{1}\right]_{2} \cup\left[s_{2}\right]_{2}$ and $J=\left[s_{3}\right]_{2} \cup\left[s_{4}\right]_{2}$. Because the elements of $S$ are pairwise non-consecutive, $|I \cap J| \leq 2$. But,

$$
|I \cap T|+|J \cap T|=|(I \cup J) \cap T|+|(I \cap J) \cap T| \leq|T|+|I \cap J| \leq 9 .
$$

Hence either $I \cap T$ or $J \cap T$ has size at most 4. Therefore either $s_{1}$ and $s_{2}$ or $s_{3}$ and $s_{4}$ are the desired elements of $S$.

Suppose now that $q \geq 3$. We prove the result by induction on $p$.

If two elements $a$ and $b$ of $S$ are consecutive (in particular if $p \leq 4 q-1$ ), then $[a]_{q} \cup[b]_{q}=2 q$, and we trivially have the result. Thus we may assume that the elements of $S$ are pairwise non-consecutive.

Assume now that an element, say $p$, is not in $S \cup T$. Then apply induction on $\mathbb{Z}_{p-1}$ with the same $S$ and $T$. It is easy to see that the two elements $a$ and $b$ such that $\left|T \backslash\left([a]_{q}^{p-1} \cup[b]_{q}^{p-1}\right)\right| \geq 3$ also satisfy $\left|T \backslash\left([a]_{q}^{p} \cup[b]_{q}^{p}\right)\right| \geq 3$.

Let $1 \leq s_{1}<s_{2}<\cdots<s_{2 q} \leq p$ be the vertices of $S$. For $1 \leq i \leq 2 q-1$, let $I_{i}=\left[s_{i}, s_{i+1}\right]$ and let $I_{2 q}=\left[s_{2 q}, p\right] \cup\left[1, s_{1}\right]$. Set $J_{i}=I_{i} \backslash S$ for each $i$. By the above arguments, each $J_{i}$ contains at least one vertex of $T$, so at most 3 vertices are in $T \cap S$. Moreover a vertex of $T$ is in exactly one $I_{i}$ if it is in $T \backslash S$ and in exactly two $I_{i}$ if it is in $T \cap S$. Hence if $q \geq 4$, there must be an $I_{i}$ such that $\left|T \cap I_{i}\right|=1$. Then $s_{i}$ and $s_{i+1}$ satisfy $\left|T \backslash\left(\left[s_{i}\right]_{q} \cup\left[s_{i+1}\right]_{q}\right)\right| \geq 3$. If $q=3$, then either there is an $I_{i}$ such that $\left|T \cap I_{i}\right|=1$ and we get the result, or $S \cap T$ is one of the two sets $\left\{s_{1}, s_{3}, s_{5}\right\}$ and $\left\{s_{2}, s_{4}, s_{6}\right\}$. In that case, one can check that $\left|T \backslash\left(\left[s_{1}\right]_{q} \cup\left[s_{2}\right]_{q}\right)\right| \geq 3$.

Proof Proof of Theorem 43 ; The proof is by induction on the number of vertices $n$. The result holds if $G$ is a triangle since there is at least $3-2=1$ choice to colour the last vertex $v$ if it is not matched, and at least $2 q+1-2 q=1$ choice to colour the last vertex $v$ if it is matched. Assume now that the result is true for every near triangulation with at most $n-1 \geq 3$ vertices, and let $G$ be a near triangulation with $n$ vertices. We let $u_{1} u_{2} \ldots u_{k}$ be the outer cycle of $G$, and $u_{1}$ and $u_{2}$ be the two precoloured vertices with respective colours $c_{1}$ and $c_{2}$.

Case 1: $G$ has a chord $u_{i} u_{j}$ with $i<j$. We use the induction hypothesis on the near triangulation whose outer cycle is $u_{1} u_{2} \ldots u_{i} u_{j} u_{j+1} \ldots u_{k} u_{1}$. Next we use the induction hypothesis on the near triangulation whose outer cycle is $u_{i} u_{i+1} \ldots u_{j} u_{i}$, the two precoloured vertices being $u_{i}$ and $u_{j}$. The result follows easily.

Case 2: $G$ has no chord. Let $v_{1}, \ldots, v_{d}$ be the neighbours of $u_{k}$ that do not belong to $C$. Without loss of generality, we can assume that $u_{k-1} v_{1} v_{2} \ldots v_{d} u_{1}$ is a path. Set $G^{\prime}=G-u_{k}$, noting that $G^{\prime}$ is a near-triangulation.

We shall distinguish four cases: 
- Subcase 1: $u_{k} u_{1} \in M$. Since $L\left(u_{k}\right) \geq 2 q+1$, there exist two colours $a$ and $b$ in $L\left(u_{k}\right) \backslash\left[c_{1}\right]_{q}$. We define the list assignment $L^{\prime}$ of $G^{\prime}$ by $L^{\prime}(v):=L(v)$ if $v \notin\left\{v_{1}, v_{2}, \ldots, v_{d}\right\}$ and $L^{\prime}(v):=$ $L(v) \backslash\{a, b\}$ otherwise. Then $\left|L^{\prime}\left(v_{i}\right)\right| \geq 2 q+3-2=2 q+1$ for each $i$. Thus we can apply the induction hypothesis to $G^{\prime}$ and $L^{\prime}$. Now we complete the colouring of $G$ by colouring $u_{k}$ with $a$ if $c\left(u_{k-1}\right) \neq a$ and with $b$ otherwise.

- Subcase 2: $u_{k-1} u_{k} \in M$. Since $L\left(u_{k}\right) \geq 2 q+1$, there exist two colours $a$ and $b$ in $L\left(u_{k}\right) \backslash\left\{c_{1}\right\}$ such that $|a-b|_{p} \geq q+1$. Thus $\left|[a]_{q} \cap[b]_{q}\right| \leq 2 q-2$. We define the list assignment $L^{\prime}$ of $G^{\prime}$ by $L^{\prime}(v):=L(v)$ if $v \notin\left\{u_{k-1}, v_{1}, v_{2}, \ldots, v_{d}\right\}$, and $L^{\prime}(v):=L(v) \backslash\{a, b\}$ otherwise. For each 3-subset $S$ of $L\left(u_{k-1}\right)$, we define the list assignment $L_{S}^{\prime}$ of $G^{\prime}$ by $L_{S}^{\prime}(v):=L(v)$ if $v \notin\left\{u_{k-1}, v_{1}, v_{2}, \ldots, v_{d}\right\}, L_{S}^{\prime}\left(u_{k-1}\right)=S$ and $L_{S}^{\prime}(v):=L(v) \backslash\{a, b\}$ otherwise. Again since $\left|L_{S}^{\prime}\left(v_{i}\right)\right| \geq 2 q+3-2=2 q+1$ for each $i$, we can apply the induction hypothesis to $G^{\prime}$ and $L_{S}^{\prime}$. Since this applies for any choice of $S$, for at least $2 q-1$ distinct elements $c$ of $L\left(u_{k-1}\right)$, there is a circular $q$-backbone $L^{\prime}$-colouring such that $u_{k}$ is assigned $c$. One of these values of $c$ is not in $[a]_{q} \cap[b]_{q}$. Hence we can extend the corresponding colouring by colouring $u_{k}$ with $a$ if $c \notin[a]_{q}$ and with $b$ otherwise, to obtain a circular $q$-backbone $L$-colouring of $G$.

- Subcase 3: $u_{k}$ is matched to $v_{j}, 1 \leq j \leq d$. Since $L\left(u_{k}\right) \geq 2 q+1$, by Lemma 44, there exist two colours $a$ and $b$ in $L\left(u_{k}\right) \backslash\left\{c_{1}\right\}$ such that $\left|L\left(v_{j}\right) \backslash\left([a]_{q} \cup[b]_{q}\right)\right| \geq 3$. We define the list assignment $L^{\prime}$ of $G^{\prime}$ by $L^{\prime}(v):=L(v)$ if $v \notin\left\{v_{1}, v_{2}, \ldots, v_{d}\right\}, L^{\prime}\left(v_{j}\right):=L\left(v_{j}\right) \backslash\left([a]_{q} \cup[b]_{q}\right)$ and and $L^{\prime}(v):=L(v) \backslash\{a, b\}$ otherwise. Then $\left|L^{\prime}\left(v_{i}\right)\right| \geq 2 q+3-2=2 q+1$ for each $i \neq j$. Thus we can apply the induction hypothesis to $G^{\prime}$ and $L^{\prime}$. Now we complete the colouring of $G$ by colouring $u_{k}$ with $a$ if $c\left(u_{k-1}\right) \neq a$ and with $b$ otherwise.

- Subcase 4: $u_{k}$ is not matched. Since $L\left(u_{k}\right) \geq 3$, there exist two colours $a$ and $b$ in $L\left(u_{k}\right) \backslash\left\{c_{1}\right\}$. We define the list assignment $L^{\prime}$ of $G^{\prime}$ by $L^{\prime}(v):=L(v)$ if $v \notin\left\{v_{1}, v_{2}, \ldots, v_{d}\right\}$ and $L^{\prime}(v):=$ $L(v) \backslash\{a, b\}$ otherwise. Then $\left|L^{\prime}\left(v_{i}\right)\right| \geq 2 q+3-2=2 q+1$ for each $i$. Thus we can apply the induction hypothesis to $G^{\prime}$ and $L^{\prime}$. Now we complete the colouring of $G$ by colouring $u_{k}$ with $a$ if $c\left(u_{k-1}\right) \neq a$ and with $b$ otherwise.

\section{Acknowledgement}

The authors would like to thank Daniel Katz for stimulating discussions and pointing out some papers leading to finding [8], and the anonymous referees for their careful reading and helpful comments.

\section{References}

[1] N. Alon. Degrees and choice numbers. Random Structures Algorithms, 16(4):364-368, 2000.

[2] N. Alon, C. McDiarmid, and B. Reed. Star arboricity. Combinatorica, 12(4):375-380, 1992.

[3] N. Alon and M. Tarsi. Colorings and orientations of graphs. Combinatorica, 12(2):125-134, 1992. 
[4] H. Broersma, F. V. Fomin, P. A. Golovach and G. J. Woeginger. Backbone colorings for networks. In Proceedings of the 29th International Workshop on Graph-Theoretic Concepts in Computer Science (WG 2003), LNCS:2880:131-142, 2003.

[5] H. Broersma, F. V. Fomin, P. A. Golovach and G. J. Woeginger. Backbone colorings for graphs: tree and path backbones. Journal of Graph Theory 55(2):137-152, 2007.

[6] H.J. Broersma, J. Fujisawa, L. Marchal, D. Paulusma, A.N.M. Salman, and K. Yoshimoto. $\lambda$ backbone colorings along pairwise disjoint stars and matchings. Discrete Mathematics 309:55965609, 2009.

[7] P. Erdôs, A. L. Rubin, and H. Taylor. Choosability in graphs. In Proceedings of the West Coast Conference on Combinatorics, Graph Theory and Computing (Humboldt State Univ., Arcata, Calif., 1979), Congress. Numer., XXVI, pages 125-157, Winnipeg, Man., 1980. Utilitas Math.

[8] R. Evans and P. Montgomery. Problem 6631. American Mathematical Monthly 98 (9): 870-872, 1991.

[9] S. L. Hakimi. On the degree of the vertices of a directed graph. J. Franklin Inst., 279:290-308, 1965.

[10] F. Havet, R. Kang, T. Müller, and J.-S. Sereni. Circular choosability. Journal of Graph Theory, 61(4):241-334, 2009.

[11] F. Havet, A. D. King, M. Liedloff, and I. Todinca. (Circular) backbone coloring: tree backbones in planar graphs. INRIA Research Report 8512, November 2012.

[12] D. Král'. Channel assignment problem with variable weights. SIAM J. Discrete Math. 20:690-704, 2006.

[13] D. D.-F. Liu and X. Zhu. Multilevel distance labelings for paths and cycles. SIAM J. Discrete Math. 19:610-621, 2005.

[14] C. McDiarmid. On the span in channel assignment problems: bounds, computing and counting. Discrete Math. 266:387-397, 2003.

[15] B. Mohar. Choosability for the circular chromatic number, 2003. http://www.fmf.uni1j.si/mohar/Problems/P0201ChoosabilityCircular.html.

[16] S. Norine. On two questions about circular choosability. Journal of Graph Theory, 58(3);261-269, 2008.

[17] S. Norine, T.-L. Wong, and Xuding Zhu. Circular choosability via combinatorial nullstellensatz. $J$. Graph Theory, 59(3):190-204, 2008.

[18] M. Tarsi. On the decomposition of a graph into stars. Discrete Math., 36(3):299-304, 1981.

[19] C. Thomassen. Every planar graph is 5-choosable. J. Combin. Theory Ser. B, 62(1):180-181, 1994.

[20] X. Zhu. Circular choosability of graphs. Journal of Graph Theory, 48(3):210-218, 2005. 